\title{
Development of linseed oil-free Bakelite Resistive Plate Chambers
}

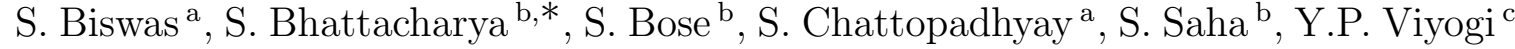

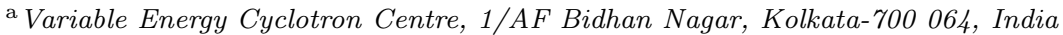 \\ b Saha Institute of Nuclear Physics, 1/AF Bidhan Nagar, Kolkata-700 064, India \\ ${ }^{\mathrm{c}}$ Institute of Physics, Sachivalaya Marg, Bhubaneswar, Orissa-751 005, India
}

\begin{abstract}
In this paper we would like to present a few characteristics of the Resistive Plate Chambers (RPC) made of a particular grade of bakelite paper laminates (P-120, NEMA LI-1989 Grade XXX), produced and commercially available in India. This particular grade is used for high voltage insulation in humid conditions. The chambers are tested with cosmic rays in the streamer mode using argon, tetrafluroethane and isobutane in 34:59:7 mixing ratio. In the first set of detectors made with such grade, a thin coating of silicone fluid on the inner surfaces of the bakelite was found to be necessary for operation of the detector. Those silicone coated RPCs were found to give satisfactory performance with stable efficiency of $>90 \%$ continuously for a long period as reported earlier. Results of the crosstalk measurement of these silicone coated RPC will be presented in this paper. Very recently RPCs made with the same grade of bakelite but having better surface finish, are found to give equivalent performance even without any coating inside. Preliminary results of this type of RPCs are also being presented.
\end{abstract}

Key words: RPC; Streamer mode; Bakelite; Cosmic rays; Silicone

PACS: 29.40.Cs

\section{Introduction}

In the proposed India-based Neutrino Observatory (INO), the RPCs [1] have been chosen as the active detector for muon detection in an Iron Calorimeter (ICAL) [2. As proposed presently, the ICAL is a sampling calorimeter consisting of 140 layers of magnetized iron, each of $60 \mathrm{~mm}$ thickness, using RPCs of $2 \mathrm{~m} \times 2 \mathrm{~m}$ area as active media sandwiched between them. A 50 kton ICAL is expected to consist of about 27000 RPC modules. For the ICAL-RPCs, main design criteria are (a) moderate position resolution $(\sim 1 \mathrm{~cm}),(\mathrm{b})$ good timing resolution $(\sim 1-2 \mathrm{~ns})$ (c) ease of fabrication in large scale with modular structure and most importantly (d) low cost. The detailed R\&D are being performed on the glass RPCs for INO 3 . A parallel effort on building and testing of the RPC modules using the bakelite obtained from the local industries in India is also going on.

At the end of nineties, it was found that the RPCs based on bakelite show serious ageing effects reducing the efficiency drastically 4]. Detailed investigations revealed that

Email address: sudeb.bhattacharya@saha.ac.in (S. Bhattacharya). the use of linseed oil for the surface treatment in such cases was the main reason for this ageing effect [5]6. Efforts were subsequently made to look for alternatives to linseed oil treatment, or to develop bakelite sheets which can be used without the application of linseed oil 7 .

The aim of the present study is to achieve stable performance of the bakelite RPC detector without linseed oil for prolonged operation. The method of construction of these RPCs and the results of the long term test has been reported earlier 8 . In this article we report some other characteristics of the silicone coated bakelite RPC and the initial results of the RPC fabricated without any coating.

\section{Design of the prototype RPCs}

The RPCs were made of two $300 \mathrm{~mm} \times 300 \mathrm{~mm} \times 2 \mathrm{~mm}$ bakelite sheets, used as electrodes, separated by a $2 \mathrm{~mm}$ gas gap. A uniform separation of the electrodes was ensured by using five polycarbonate button spacers of $10 \mathrm{~mm}$ diameter and $2 \mathrm{~mm}$ thickness, and edge spacers of $300 \mathrm{~mm} \times 8 \mathrm{~mm}$ $\times 2 \mathrm{~mm}$ dimension. Two polycarbonate made nozzles were used for gas inlet and outlet 910.

The high voltages (HV) to the RPC were applied on the graphite coating (surface resistivity $\sim 1 \mathrm{M} \Omega / \square$ ) made 


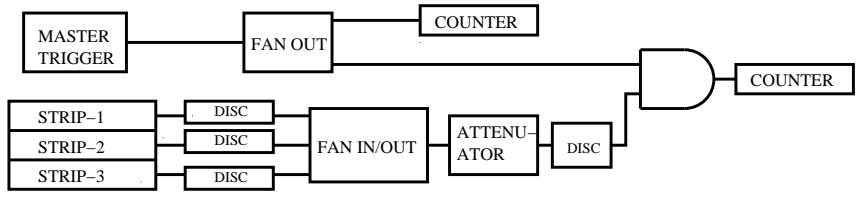

Fig. 1. Schematic representation of the crosstalk measurement setup.

over the outer surfaces of the bakelite. The induced RPC signals were collected using copper and foam based pick-up strips, each of area $300 \mathrm{~mm} \times 25 \mathrm{~mm}$ with a separation of $2 \mathrm{~mm}$ between two adjacent strips. The pick-up strips were covered with $100 \mu \mathrm{m}$ thick kapton foils to isolate them from the graphite layers.

Premixed gas of Argon, Isobutane and Tetrafluroethane (R-134a) was used in 34:7:59 mixing ratio. A typical flow rate of $0.4 \mathrm{ml}$ per minute was maintained by the gas delivery system, 11, resulting in $\sim 3$ changes of gap volume per day.

\section{Cosmic ray test setup}

The RPCs were tested in the same cosmic ray test bench described in Ref. 6. The coincidence between the signals obtained from the scintillator I $(350 \mathrm{~mm} \times 250 \mathrm{~mm}$ size $)$, the finger scintillator(III) $(200 \mathrm{~mm} \times 40 \mathrm{~mm}$ size $)$ which was placed above the RPC plane and the scintillator II (350 $\mathrm{mm} \times 250 \mathrm{~mm}$ size) which was placed below, was taken as the Master trigger. Finally, the ORed signal obtained from two adjacent pick-up strips of the chamber was put in coincidence with the master trigger obtained above. This was referred to as the coincidence trigger of the RPC. The efficiency of the RPC detector, taken as the ratio between the coincidence trigger rates of the RPC and the master trigger rates of the 3 -element plastic scintillator telescope was measured over an area $200 \mathrm{~mm} \times 40 \mathrm{~mm}$ which was the window of the cosmic ray telescope. The average master trigger rate was $\simeq 0.005 \mathrm{~Hz} / \mathrm{cm}^{2}$.

Fig. 1 shows the schematic of the crosstalk measurement setup. The width of the finger scintillator covered one pickup strip completely and two adjacent strips partially. The signals from these three strips after leading edge discriminator (LED threshold : $40 \mathrm{mV}$ ) were sent to the input of a fan in module. The output signal of the previous stage after attenuation was again put to another discriminator. The signal from this discriminator was taken in coincidence with the master trigger. The crosstalk was defined by the ratio of this coincidence count and the master trigger. The attenuation factor was set at 0.3 . The fan out signal after attenuation, observed in the oscilloscope, was $\sim 250 \mathrm{mV}$ when one strip fires. The resulting pulse heights were $\sim 440$ $\mathrm{mV}$ and $630 \mathrm{mV}$ respectively, when signals from two strips and three strips came simultaneously. In order to measure the crosstalk between two and three adjacent strips, the discriminator threshold to the fan out signal was set at 280 $\mathrm{mV}$ and $520 \mathrm{mV}$, respectively.

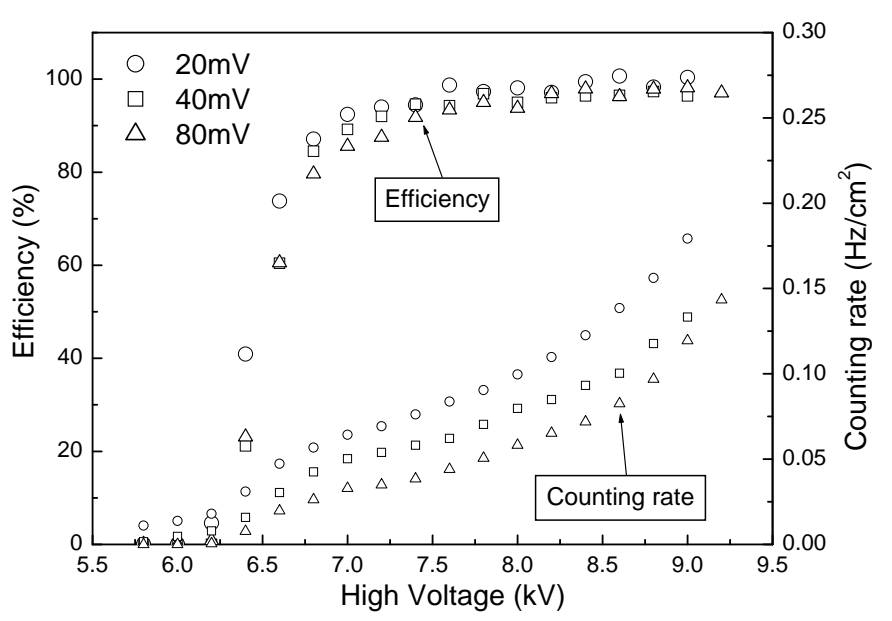

Fig. 2. The efficiency and the counting rate as a function of high voltage for a silicone coated RPC made of P-120 grade bakelite with discriminator threshold values of $20 \mathrm{mV}, 40 \mathrm{mV}$ and $80 \mathrm{mV}$. The gas mixture used was Argon (34\%) + Isobutane (7\%) + R-134a (59\%).

\section{Results}

The following properties have been studied in the test setup for all the chambers: (a) the efficiency of the chambers and their variation with change in different parameters e.g. HV, gas composition, laboratory environment etc., (b) the variation of the counting rate, (c) the leakage current and their variation and (d) the long term stability in the streamer mode. The long term behavior of the silicone coated RPCs in the streamer mode has been reported earlier 10. Some more results are presented in this section.

The efficiency and the counting rate, which is also known as the noise rate, of the RPCs have been studied by varying the applied HV and setting different discriminator threshold values. The variation of the efficiency and the counting rates with the applied HV for three discriminator threshold value of a silicone coated RPC is shown in Fig. 2. It is seen that the counting rate of the RPC decreases with the increase of the threshold value which is expected with the suppression of noise at a higher threshold value. However, the efficiency curves do not depend much on the threshold setting varying between $20 \mathrm{mV}$ to $80 \mathrm{mV}$, except that the efficiency plateau is marginally higher at the lowest threshold setting of $20 \mathrm{mV}$. A typical screen dump of the oscilloscope pulse at $8 \mathrm{kV}$ is shown in Fig. 3. The figure shows the rise time is about $6 \mathrm{~ns}$, which indicates the fastness of the pulse.

The effect of the environmental humidity on the efficiency curves has also been studied. This measurement was done at relative humidities of $58 \%$ and $67 \%$ of the laboratory environment and at the room temperature of $\sim 22-25^{\circ} \mathrm{C}$. These curves, plotted in Fig. 4, indicate no effect of humidity on the efficiency. However, the counting rate (shown in Fig. 4) and the leakage currents, measured simultaneously and plotted in Fig. 5, are larger at higher humidity. These 


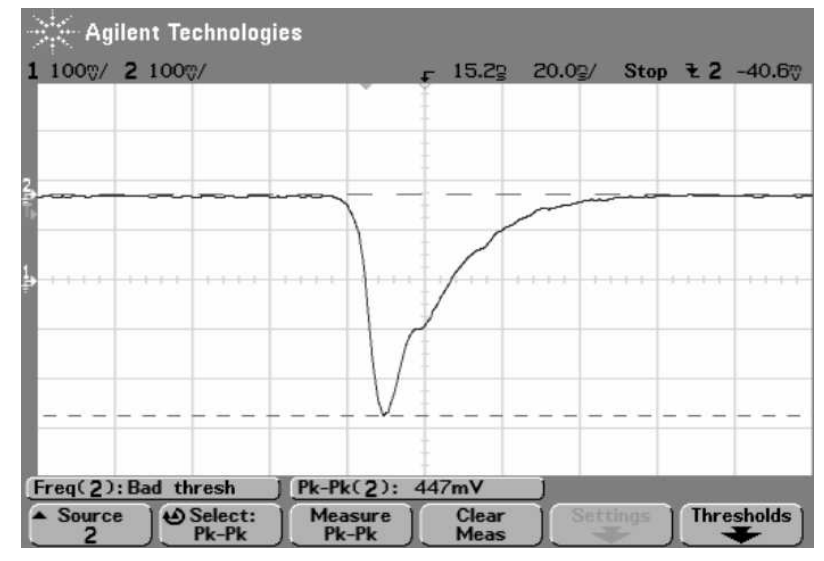

Fig. 3. Average induced pulse on a pick-up strip at $8 \mathrm{kV}(100 \mathrm{mV} /$ Div, $20 \mathrm{~ns} /$ Div, $50 \Omega$ load) of a silicone coated P-120 grade bakelite RPC.

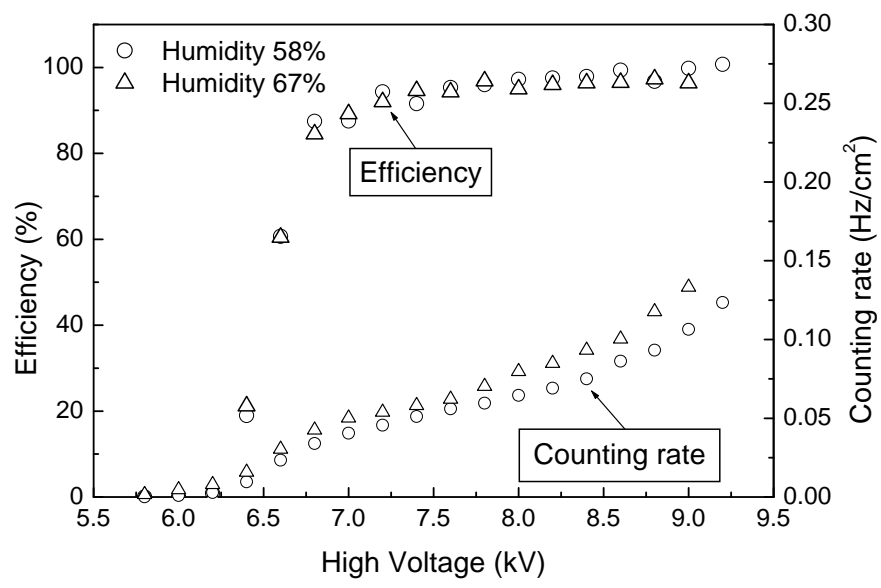

Fig. 4. The efficiency and the counting rate versus high voltage for different humidities for a silicone coated P-120 grade bakelite RPC.

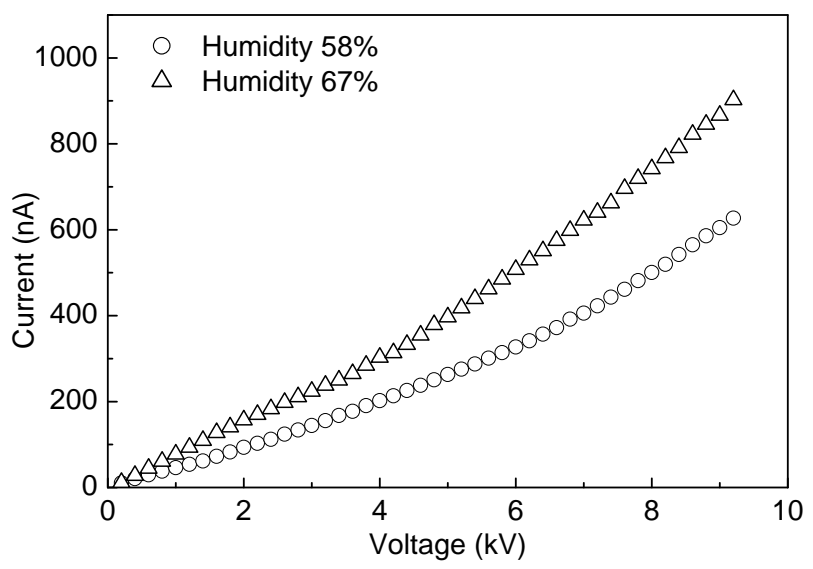

Fig. 5. Current versus high voltage for different humidities for a silicone coated P-120 grade bakelite RPC.

observations indicate that charge leakage through the exterior surfaces may be contributing more at higher humidity.

The long cable drive of RPC streamer pulse has been

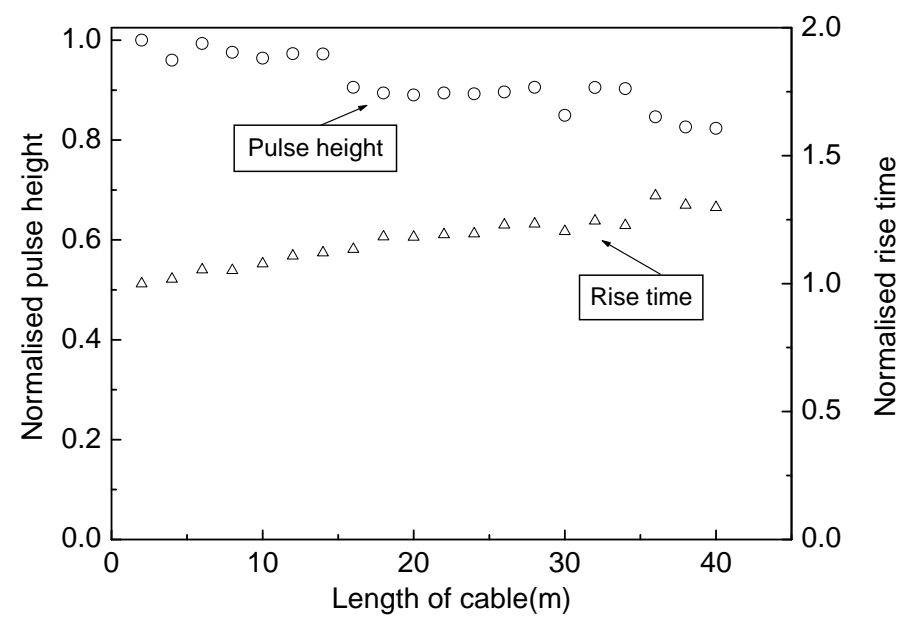

Fig. 6. Variation of the normalised pulse height and rise time with the cable length for a silicone coated P-120 grade bakelite RPC.

tested using RG-174/U coaxial cables. A maximum of $40 \mathrm{~m}$ RG-174/U coaxial cable has been used. The average pulse height of RPC in streamer mode was $\sim 300-500 \mathrm{mV}$ as shown in Fig. 3. The signal amplitude was attenuated to $\sim$ $80 \%$ after $40 \mathrm{~m}$ cable drive. The rise time increases slightly at that length of cable. The variation of normalised pulse height and rise time are shown in Fig. 6.

The measurement of the crosstalk (CT) for a silicone coated RPC was carried out rigorously. When a single particle induces signal on two or more strips then the term crosstalk comes into the picture. The crosstalk between the two and three RPC strips, defined in Section 3, had been measured by varying the applied HV and is shown in Fig 7 . When the discrimination threshold after attenuation was set at $280 \mathrm{mV}$ as stated in Section 3, the signals coming from the two adjacent strips as well as three adjacent strips simultaneously contributed to the crosstalk and it was found to be $<20 \%$ (Fig 7 ). The crosstalk between the three adjacent strips was found to be $<5 \%$. These values of the crosstalk have been taken into account while estimating the final efficiency.

The time resolution of a silicone coated RPC was measured and it was found to be $\sim 2$ ns (FWHM of the time spectra) at $8 \mathrm{kV}$. The variation of time resolution with $\mathrm{HV}$ of that particular detector is shown in Fig. 8.

Finally, one module was made with $1.6 \mathrm{~mm}$ thick P-120 grade bakelite sheets with better surface finish. No coating was applied to that RPC. The efficiency curve and the variation of the counting rate with $\mathrm{HV}$ is plotted in Fig. 9. The current for this particular RPC was found to be higher than that of the silicone coated one. It was about $2 \mu \mathrm{A}$ at $8 \mathrm{kV}$.

\section{Conclusions and outlook}

In conclusion, a rigorous study of RPCs made from a particular grade of bakelite commercially available in India has been performed. An efficiency of $>90 \%$ is obtained 


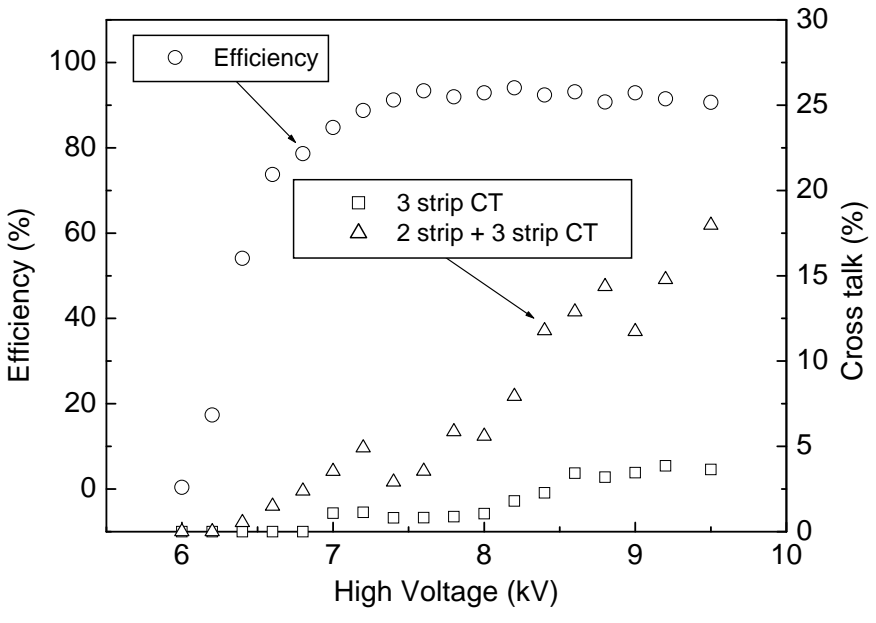

Fig. 7. Variation of the crosstalk with the high voltage in comparison with the efficiency for a silicone coated P-120 grade bakelite RPC.

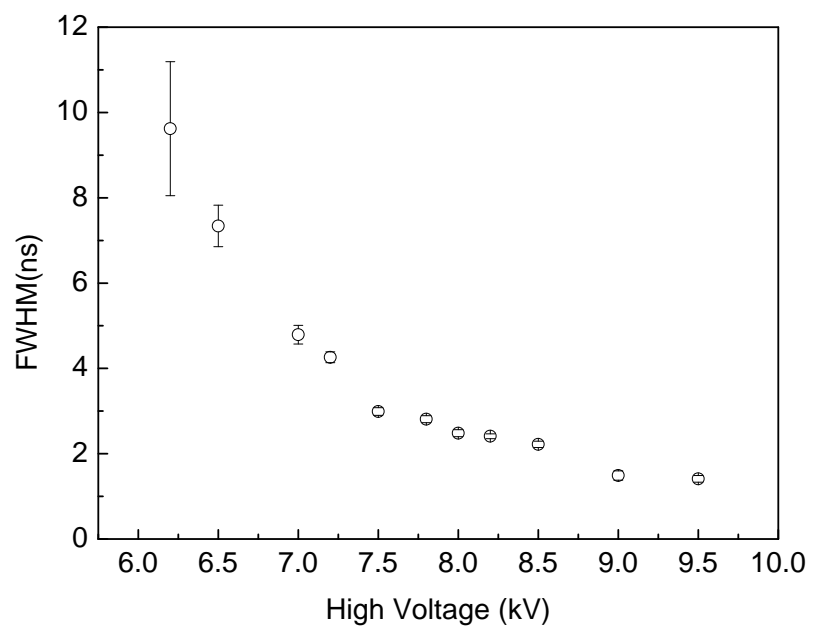

Fig. 8. The time resolution as a function of $\mathrm{HV}$ for a silicone coated P-120 grade bakelite RPC.

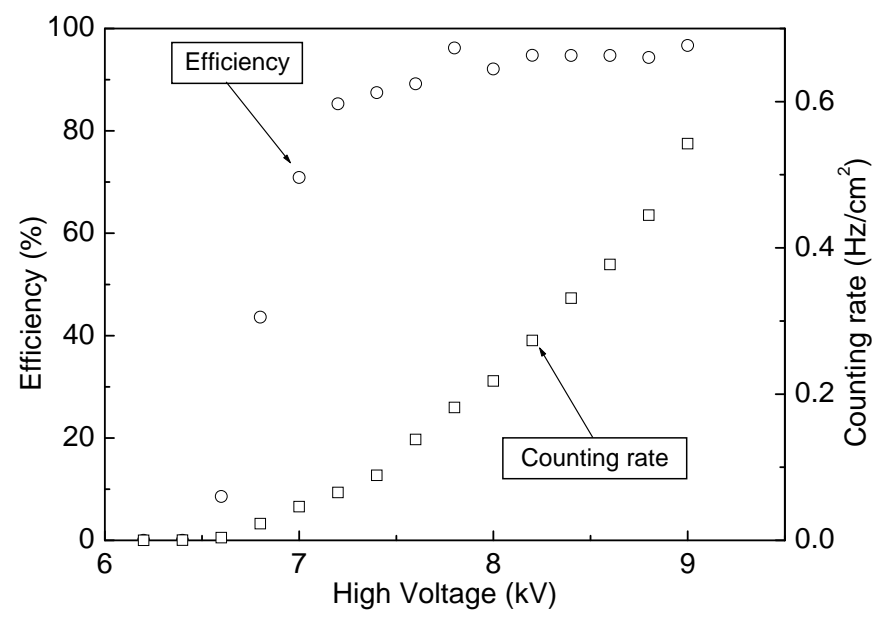

Fig. 9. The efficiency and the counting rate as a function of HV for uncoated P-120 grade bakelite RPC. for silicone coated P-120 grade bakelite RPC. The effect of threshold and external humidity on the performance of the RPCs are presented in this paper. The current as well as the counting rate increases with the increase of environmental humidity.

The RPC streamer signals can be driven for a long distance without any significant attenuation. The crosstalk between two neighboring strips (which may be due to some real event) is found to be about $15 \%$ and that between three neighboring strips is about $5 \%$. The measured time resolution of a particular silicone coated RPC is found to be $\sim 2$ ns which is comparable to any single gap glass or linseed oil coated bakelite RPC. Lastly the preliminary data of a uncoated RPC is reported which show encouraging results, as one can plan for large RPC $(1 \mathrm{~m} \times 1 \mathrm{~m})$ with this particular grade of bakelite with better surface finish and without any coating.

Further studies in this direction are being carried out which include timing measurements of silicone coated RPCs and performance of oil-less RPCs. These will be reported at a later stage.

\section{Acknowledgement}

We are thankful to Prof. Naba Kumar Mondal of TIFR, India and Prof. Kazuo Abe of KEK, Japan for their encouragement and many useful suggestions in course of this work. We acknowledge the service rendered by Mr. Ganesh Das of VECC for meticulously fabricating the detectors. We would like to thank the SINP and VECC HEP workshop for making the components of the detectors. Finally we acknowledge the help received from the scientific staff of Electronics Workshop Facility of SINP for building the gas flow control and delivery system of the gas mixing unit used in this study.

\section{References}

[1] R. Santonico, R. Cardarelli, Nucl. Inst. and Meth. 187, (1981) 377 .

[2] INO Project Report, INO/2006/01, June 2006, $\langle$ http://www.imsc.res.in/ ino/〉

[3] N.K. Mondal, Talk given at IX International Workshop on Resistive Plate Chambers and Related Detectors, Mumbai, February 2008.

[4] J. Va'vra, Nucl. Inst. and Meth. A 515 (2003) 354.

[5] F.Anulli et al., Nucl. Inst. and Meth. A 508 (2003) 128.

[6] F. Anulli et al., Nucl. Inst. and Meth. A 515 (2003) 322.

[7] J. Zhang et al., Nucl. Inst. and Meth. A 540 (2005) 102.

[8] S. Biswas, Talk given at IX International Workshop on Resistive Plate Chambers and Related Detectors, Mumbai, February 2008.

[9] S. Biswas et al., arXiv:0802.2766

[10] S. Biswas et al., Proceedings of IX International Workshop on Resistive Plate Chambers and Related Detectors, Mumbai, February 2008.

[11] S. Bose et al., Proceedings of IX International Workshop on Resistive Plate Chambers and Related Detectors, Mumbai, February 2008 . 